\title{
Heat Capacity and Thermal Damping Properties of Spin- Crossover Molecules: a New Look at an Old Topic
}

\author{
Karl Ridier, ${ }^{[a]}$ Yuteng Zhang, ${ }^{[a]}$ Mario Piedrahita-Bello, ${ }^{[a]}$ Carlos M. Quintero, ${ }^{[a, b]}$ Lionel Salmon, ${ }^{[a]}$ Gábor \\ Molnár, ${ }^{*[a]}$ Christian Bergaud, ${ }^{[b]}$ and Azzedine Bousseksou ${ }^{*[a]}$
}

Abstract: The thermally-induced spin-crossover phenomenon in transition metal complexes is an entropy driven process, which has been extensively studied through calorimetric methods. Yet, the excess heat capacity associated with the molecular spin-state switching has never been explored for practical applications. Herein, we experimentally assess the thermal damping effect induced by a spin-crossover film on the transient heating response of metallic microwires, Joule-heated by current pulses. A damping of the wire temperature, up to $10 \%$, is evidenced on a time scale of tens of microseconds due to the spin-state switching of the molecular film. We demonstrate fast heat-charging dynamics and negligible fatigability which, together with the solid-solid nature of the spin transition, appear as promising features for achieving thermal energy management applications in functional devices.

Molecular spin-crossover (SCO) complexes of certain transition metal ions, which display a reversible transformation between the so-called low-spin (LS) and high-spin (HS) states, constitute an archetypal example of multifunctional, switchable molecular materials. ${ }^{[1-3]}$ The spin-state switching can be triggered by a variety of external stimuli (temperature or pressure change, light irradiation, etc.) and involves spectacular changes in the optical, magnetic, electrical and mechanical properties. These attributes, coupled to recent progress in the nanoscale synthesis, pave the way for the integration of these molecular switches in sensors, actuators and other devices. ${ }^{[4-6]}$ Another interesting property of SCO materials concerns the endothermic/exothermic character of the spin transition, ${ }^{[1,7]}$ i.e. the possibility of absorbing/releasing reversibly a certain amount of thermal energy with the spin-state switching. The applicative potential of this thermal property of SCO complexes, in terms of thermal management, has never been explored in a functional device, though it has been recently evoked for barocaloric refrigeration purposes. ${ }^{[8,9]}$

The issue of thermal energy storage and/or thermal management constitutes a present-day challenge in various fields such as construction industry, renewable energy technologies, biomedical-carrying systems, etc. ${ }^{[10]}$ In particular, thermal management has become an increasingly important focus for the microelectronics industry. Indeed, miniaturization of (opto)electronic components, together with increased density and high-frequency operation, have the effect of considerably increasing the heat fluxes, possibly leading to localized transient overheating phenomena, whose consequences can range from a performance reduction to an irreversible deterioration. ${ }^{[11]}$ Heat fluxes of the electronic chips are typically approaching 300 W.cm 2 in the near future, ${ }^{[12]}$ while the maximal allowable temperature of various electronic components generally ranges between $85^{\circ} \mathrm{C}$ and $120^{\circ} \mathrm{C} \cdot{ }^{[13]}$

In this context, thermal management technologies based on latent heat storage incorporating phase-change materials (PCMs) represent appealing and efficient alternatives. ${ }^{[14]}$ Beyond the relatively small energy absorption associated with their "normal" heat capacity (sensible heat), PCMs have the capabilities of charging/discharging latent heat, in quasi-isothermal conditions, due to the excess heat capacity accompanying the phase transition. The most widely used PCMs are those involving a solid-liquid phase transition (paraffins, fatty acids, salt hydrates), associated with a comparatively large melting enthalpy (latent heat of fusion), typically above $150 \mathrm{J.g}^{-1} \cdot{ }^{[15]}$ However, the use of solid-liquid PCMs involves important practical challenges in terms of fabrication complexity and integration (e.g. encapsulation). In this context, solid-solid PCMs have received growing interest because of their inherent advantages over solid-liquid counterparts (leakage-free, smaller volume change, greater design flexibility, etc.), even though they generally exhibit smaller latent heats. ${ }^{[16]}$

Here, we postulate that SCO compounds represent a novel, promising class of solid-solid phase-change materials for thermal management applications. Not only the enthalpy change $(\Delta H)$ associated with the spin-state switching event is substantial (typically in the range 20-60 J.g-1 for above-room-temperature spin transitions ${ }^{[17]}$ ), but SCO materials have the advantage of a reversible, solid-solid transition with intrinsically fast (sub- $\mu \mathrm{s}$ ) switching times near room temperature. In this study, we have experimentally assessed the thermal damping effect of molecular films of the SCO complex [ $\left.\left.\mathrm{Fe}\left(\mathrm{HB}(\mathrm{tz})_{3}\right)_{2}\right)\right]$ (tz = 1,2,4-triazol-1-yl) 1 in a functional device. Notably, we evidence a transient attenuation of the temperature rise of SCO-coated metallic microwires, occurring on a time scale of tens of microseconds, in response to sharp electro-thermal solicitations. This work thus provides a first milestone toward the assessment of the potential of SCO materials for thermal management applications.

Compound $\mathbf{1}$ is a molecular SCO complex (Figure 1a), ${ }^{[18]}$ which can be deposited in the form of high-quality, large-area, thin films by vacuum thermal evaporation. ${ }^{[19]}$ The resulting nanocrystalline 
films are known to be homogenous, stable, and they display a fully complete and abrupt spin transition around $T_{s c O}=64^{\circ} \mathrm{C}$ (Figures 1b-1d). Calorimetric data recorded for the bulk powder of 1 show that the spin-state switching is associated with sharp, well-defined excess heat capacity peaks (Figures 1e-1f). The corresponding

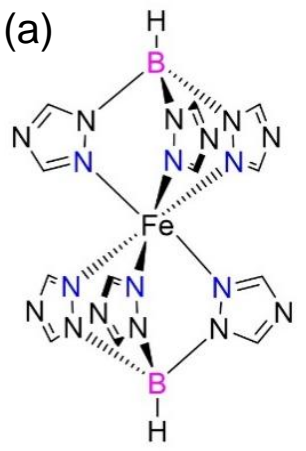

(b)
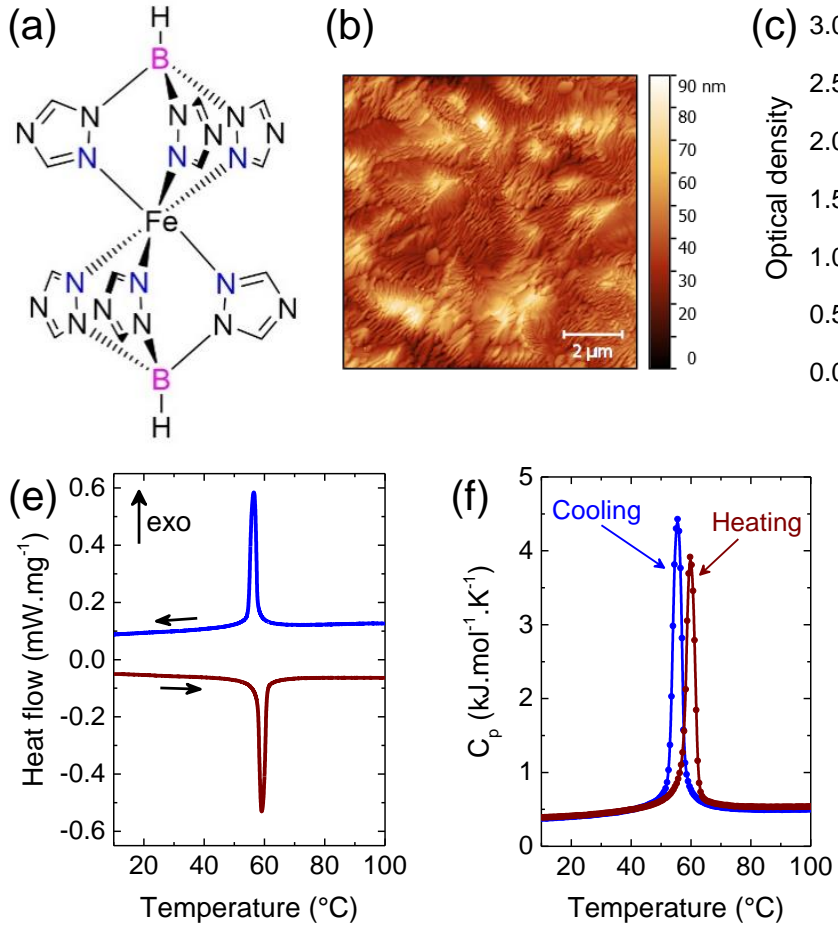

enthalpy and entropy changes are $\Delta H=19 \mathrm{~kJ} \mathrm{~mol}^{-1}\left(40 \mathrm{~J}^{-1}{ }^{-1}\right)$ and $\Delta S=57 \mathrm{~J} \cdot \mathrm{mol}^{-1} \cdot \mathrm{K}^{-1}$, respectively. Another remarkable characteristic of compound $\mathbf{1}$ is the strong robustness of the spin transition and the exceptionally high resilience of the material upon extended thermal cycling in ambient air. ${ }^{[20]}$

Figure 1. (a) Molecular structure of the complex 1. (b) Atomic force microscopy (AFM) topography image of a 900-nm-thick film of 1. (c) Optical absorbance spectra of the film acquired at different selected temperatures in the heating mode between $20^{\circ} \mathrm{C}$ (LS state) and $120^{\circ} \mathrm{C}$ (HS state). (d) Temperature dependence of the fraction of $\mathrm{HS}$ molecules extracted from the variation of the optical density. (e) Differential scanning calorimetry (DSC) analysis of the bulk powder of $\mathbf{1}$ acquired at a scan rate of $2^{\circ} \mathrm{C} \cdot \mathrm{min}^{-1}$. (f) Thermal evolution of the molar heat capacity $\left(C_{p}\right)$ of 1 , showing the excess $C_{p}$ associated with the spin-transition phenomenon. (g) Scanning electron microscopy (SEM) image of a 2-mm-long, 2- $\mu$ m-wide gold wire used as a micro-heater on a glass substrate.

To assess the thermal protection effect of molecular films of $\mathbf{1}$, Joule-heated gold microwires (Figure 1g) were fabricated on top of glass and oxidized silicon wafers (Figure S1) by conventional lithography techniques (see details in the Supporting Information, SI). A 900-nm-thick film of $\mathbf{1}$ was then deposited on top of the chip by vacuum thermal evaporation, as described recently. ${ }^{[21]}$ It is important to mention that both the deposition conditions as well as the physical properties of the SCO film are not invasive and do not modify the electrical performance of the device. The SCOcoated chip was then mounted on a variable-temperature stage to control the temperature of the entire substrate. This latter, which differs from the temperature of the heating wire $T_{\text {wire, }}$, will be hereafter denoted "base temperature" $T_{b}$. These micro-wire heaters are known to be particularly interesting for their reliable all-electrical operation and their ability to generate fast, localized heating ( $T$-jumps) with low-power excitations. ${ }^{[22]}$ In particular, the thermal variation induced by the applied current generates a delta of electrical resistance $\Delta R$, proportional to the average temperature rise of the wire $\left(\Delta R=\alpha \Delta T_{\text {wire }}\right)$, making it possible to characterize their temperature in real time, electrically (Figure S2). To demonstrate the thermal damping effect of the film of $\mathbf{1}$, deposited on top of the micro-heater, our experiment consists in sending sharp current steps in the wire and monitoring its transient heating response with a sub- $\mu$ s time scale resolution. To this aim, we used a custom-built differential resistance measurement setup (Figure S3), which simultaneously sends the input current to the wire under study ( $\left.R_{\text {wire }}\right)$ and to a reference resistance $\left(R_{r e f}\right)$ of identical value as the wire (at low-bias currents), but with a negligible temperature dependence. Since $R_{\text {ref }}$ remains unchanged independently of the current, the output signal of the circuit $V_{\text {out }}=G\left(V_{\text {wire }}-V_{\text {ret }}\right)$, where $G$ is the gain, simply corresponds to a scaled version of the temperature rise $\Delta T_{\text {wire }}$ induced in the wire due to Joule heating ${ }^{[23]}$ (see details in Figure S3).

Figure $2 \mathrm{a}$ depicts the time evolution of the temperature rise $\Delta T_{\text {wire }}$ of a gold microwire $\left(R=98.7 \Omega\right.$ and $117.8 \Omega$ at $T_{b}=25^{\circ} \mathrm{C}$ and $95^{\circ} \mathrm{C}$, respectively) deposited on top of a glass substrate, induced by a sharp current step of $20 \mathrm{~mA}$. These measurements were performed at selected base temperatures of the substrate from $25^{\circ} \mathrm{C}$ to $95^{\circ} \mathrm{C}$. Each $\Delta T_{\text {wire }}$ curve is the result of measurements averaged over 512 current steps, guaranteeing an excellent accuracy and, at the same time, proving the reproducible character of the observed phenomena. Another way to represent the data of Figure 2a consists in plotting $\Delta T_{\text {wire }}$ as a function of the base temperature at different times, as displayed in Figure $2 \mathrm{~b}$. Far from the spin-transition temperature ( $\left.T_{s c o}\right), \Delta T_{\text {wire }}$ follows a linear evolution as a function of the base temperature, slightly increasing with $T_{b}$, in agreement with the electrical characterizations of the wire performed in the steady state (Figure S2). Interestingly, for temperatures slightly below $T_{s c o}$, we observe a clear inflection of 
$\Delta T_{\text {wire, }}$ which goes through a minimum, signifying that the temperature rise experienced by the wire is transiently reduced

(a)

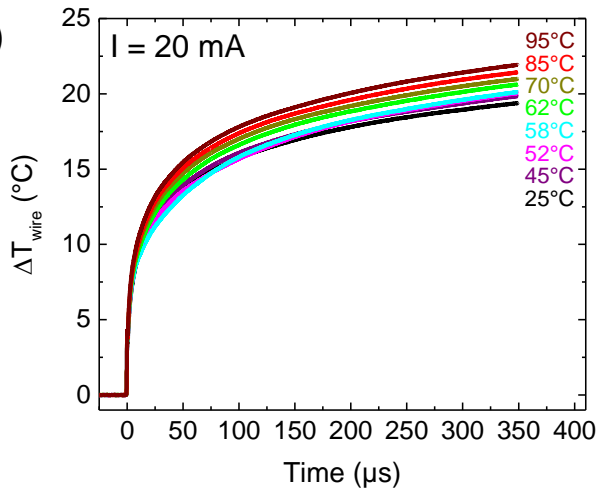

(c)

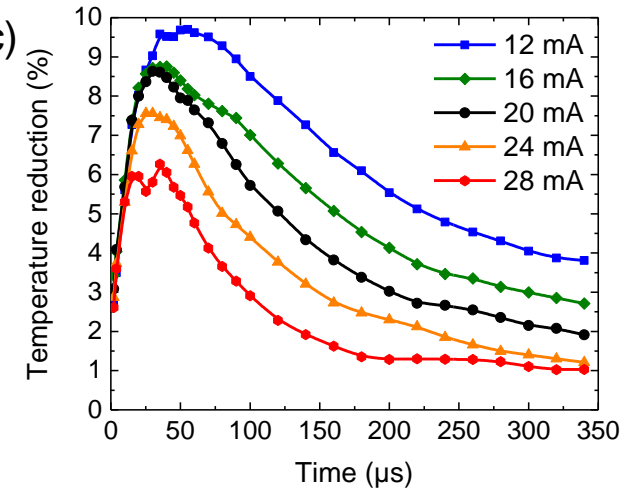

due to the SCO phenomenon, because a part of the thermal energy was absorbed following the LS-to-HS switching.
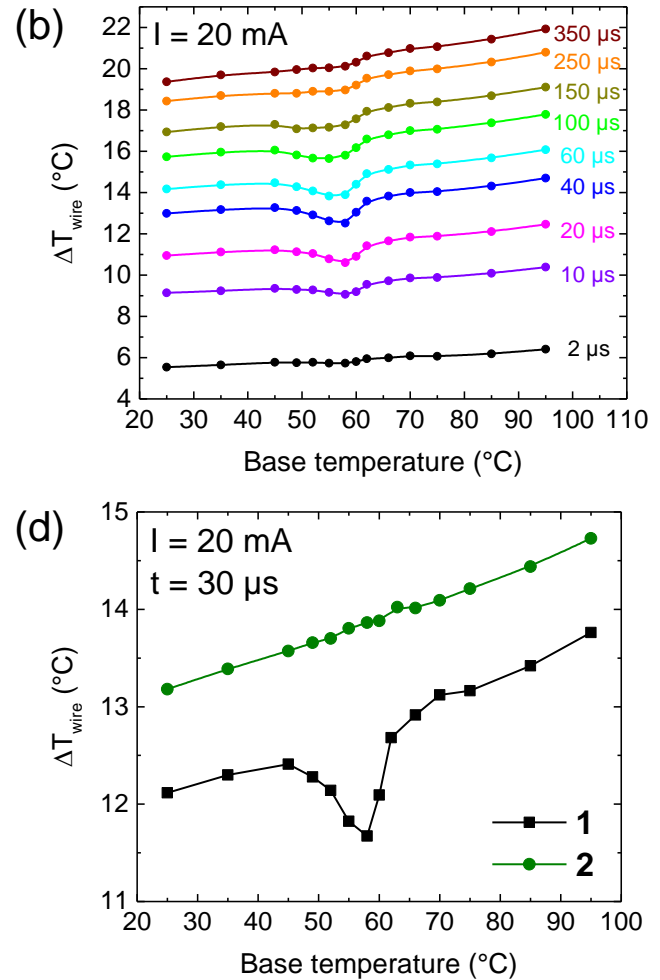

Figure 2. (a) Time evolution of the temperature rise $\Delta T_{\text {wire }}$ of a gold microwire (on top of a glass substrate) coated with a 900-nm-thick film of 1. The wire was Jouleheated by the application of a sharp current step of $20 \mathrm{~mA}$ (at $t=0$ ) at different base temperatures $T_{b}$ of the substrate. (b) Evolution of $\Delta T_{\text {wire }}$ as a function of $T_{b}$ at different selected times. (c) Time evolution of the percentage of temperature reduction $\kappa(\%)$ in the wire, following the application of a sharp current step of different intensities. (d) Comparative evolution of $\Delta T_{\text {wire }}$ with $T_{b}$, at $t=30 \mu \mathrm{s}$, following the application of a current step of $20 \mathrm{~mA}$, when the microwire is coated with a 900 $\mathrm{nm}$-thick film of $\mathbf{1}$ and a 430 -nm-thick film of the Zn-substituted complex 2.

Figure $2 \mathrm{c}$ shows the maximum percentage of temperature reduction $(\kappa)$ of the wire as a function of time, owing to the heat absorption by the SCO film. This quantity is defined as the ratio between the amplitude of the "negative" peaks observed in Figure $2 \mathrm{~b}$, arising from the SCO, and the expected value of $\Delta T_{\text {wire }}$ that would be observed in the absence of SCO, given by the linear baseline. In Figure 2c, the time evolution of the ratio $K$ is displayed for different excitation currents in the wire, ranging from $12 \mathrm{~mA}$ to $28 \mathrm{~mA}$. The first observation is that, for a given current, the temperature damping effect goes through a maximum before progressively decreasing with time. As an example, applying a current of $20 \mathrm{~mA}$, the SCO film provides its optimum benefits in terms of heating reduction after $30 \mu \mathrm{s}$, where the temperature rise of the wire is reduced by $8.6 \%$. For longer times, the damping effect is progressively attenuated, certainly, because an increasing fraction of the SCO film is already transformed into the HS state and becomes consequently inactive, while the system continually approaches the steady-state. Interestingly, the optimum efficiency in terms of temperature reduction is not instantaneous, but only occurs after several tens of $\mu \mathrm{s}$. This delay cannot be attributed to the thermally activated switching dynamics of the SCO molecules, neither to the effect of heat propagation within the SCO layer, which are faster phenomena in thin films. ${ }^{[24]}$ Instead, this observation is likely related to the fact that the thermal spin transition in the films of $\mathbf{1}$ is not perfectly abrupt. Indeed, as $80 \%$ of the transition is spanned over a temperature range of ca. $12{ }^{\circ} \mathrm{C}$, a heating of at least ten degrees must be reached to effectively exploit the heat storage capacities of the SCO film.

As depicted in Figure 2c, another important observation is that the percentage of temperature reduction strongly depends on the excitation current. An optimal effect is obtained for the lowest excitation (12 $\mathrm{mA})$, for which the wire temperature is found to be attenuated by $9.7 \%$ after $50 \mu \mathrm{s}$. On the other hand, the temperature reduction does not exceed $6 \%$ for a current step of $28 \mathrm{~mA}$. This effect arises from the fact that the increased heat flux $\delta q$ generated by the wire is not counterbalanced by the limited heat absorption rate of the SCO film, which results in minimizing the amplitude of the thermal damping effect for large excitation currents.

By way of comparison, an identical experiment was carried out on a similar gold microwire on top of a glass substrate, but this time coated with a 430-nm-thick film of the isomorphous, $\mathrm{Zn}$ substituted complex $\left[\mathrm{Zn}\left(\mathrm{HB}(\mathrm{tz})_{3}\right)_{2}\right] 2$. This complex, which is obviously SCO inactive, is an interesting, comparative system to unambiguously demonstrate the actual effect induced by the SCO phenomenon. Predictably, as depicted in Figure $2 \mathrm{~d}$ for compound 2, $\Delta T_{\text {wire, }}$ measured $30 \mu \mathrm{s}$ after the application of the current, follows a perfectly linear behavior in the whole range of $T_{b}$, providing further evidence that the temperature reduction effect, observed with the film of $\mathbf{1}$, exclusively originates from the spintransition phenomenon (see also Figure S4). Finally, we have also conducted experiments with the same SCO-coated microwires on top of a silicon substrate. As shown in Figure S5, only a smal 
inflection of $\Delta T_{\text {wire }}$ is observed at the spin-transition temperature and the maximum percentage of temperature reduction does not exceed $2 \%$. In this particular case, due to its large thermal resistance compared to silicon, the SCO film has only a minor influence on the temperature rise of the wire.

Overall, these first proof-of-concept experiments demonstrate that temperature rise in microcircuits can be effectively damped by the excess heat capacity of a solid-solid spin-transition material. In particular, the fast heat-charging kinetics appears particularly promising for attenuating temperature spikes, which might accidentally arise. Importantly, the heat storage property of our SCO-based thermal damper was found to be fully preserved after thousands of heating solicitations in ambient air. These first quantitative experiments open up novel fields of application in SCO research, with particular relevance for the emerging areas of organic and flexible electronics. The vast library of SCO materials should allow not only for custom-designed applications, but also offers unique opportunities to develop innovative solutions that combine energy storage and/or thermal management with other functional attributes. ${ }^{[4,5]}$ Obviously, the simple configuration of the present device is not particularly favorable to achieve high temperature reduction. Yet, this set-up provides a straightforward starting point for finite element simulations and for the development of more powerful engineering strategies. An interesting approach for a more efficient thermal damping effect, would be the inclusion of the SCO material directly into cavities of the heat-sink substrate, offering the double ability of efficiently dissipating heat and absorbing a part of it into the phase-change enthalpy. ${ }^{[25]}$ On the other hand, the incorporation of a thin metal film within (or on top of) the SCO layer could help to spread the heat dissipation along in-plane directions, resulting in an increased thermal exchange surface. ${ }^{[26]}$ The issue of low thermal conductivity, which constitutes a recurrent impediment for most PCMs, may be also addressed through the dispersion of high-conductivity nanostructures (carbon nanotubes, metallic particles, etc.) into the SCO material[27] or, vice-versa, by incorporating SCO particles in a porous metal foam or other high-thermal-conductivity matrices. ${ }^{[28]}$ At the same time, it appears necessary to substantially increase the amount of SCO material. Indeed, considering the mean heat flux generated by our microwire $\left(P_{S}\right.$ $400 \mathrm{~W} . \mathrm{cm}^{-2}$ for $I=20 \mathrm{~mA}$ ) - which corresponds to those typically observed in electronic chips ${ }^{[12,25]}$ - as well as the physical properties of the SCO film (mass density $\rho=1.5 \mathrm{~g} . \mathrm{cm}^{-3}$, transition enthalpy $\Delta H=40 \mathrm{~J}^{-1} \mathrm{~g}^{-1}$ and thickness $e=900 \mathrm{~nm}$ ), a simplified calculation can provide a rough estimation of the duration of the thermal damping effect $\Delta t=(\rho \Delta H \times e) / P_{S}=14 \mu \mathrm{s}$. This time scale is in agreement with our experimental observations. Following the same approach, a film thickness of ca. $100 \mu \mathrm{m}$ would be, for instance, necessary to get sufficient mass of SCO to absorb the required amount of heat during typical power transients of $1 \mathrm{~ms}$. For such large thicknesses, our vacuum thermal evaporation method is not favorable (too slow) and novel deposition techniques (printing, coating, etc.) will have to be developed.

\section{Acknowledgements}

We thank Denis Lagrange (LAAS-CNRS) for his help with the differential resistance set-up. The PhD grants of $Y$. Z. and M. P.B. were financed by the China Scholarship Council and the
Federal University of Toulouse/Occitanie Region, respectively. This work was supported by the Micro and Nanotechnologies Platform of LAAS-CNRS (Toulouse, France), which is a member of the French RENATECH Network.

Keywords: Thermal management $\cdot$ Thermal energy storage $•$ Spin crossover $\cdot$ Molecular thin film $•$ Excess heat capacity

[1] P. Gütlich, A. Hauser, H. Spiering, Angew. Chem., Int. Ed. Engl. 1994 33, 2024.

[2] Spin-Crossover Materials: Properties and Applications (Ed.: M. A Halcrow), John Wiley \& Sons, Ltd., New York, 2013.

[3] Spin crossover phenomenon (Ed.: A. Bousseksou) C. R. Chim. 2018, 21, pp. 1055-1300.

[4] G. Molnár, S. Rat, L. Salmon, W. Nicolazzi, A. Bousseksou, Adv. Mater 2018, 30, 1703862.

[5] K. Senthil Kumar, M. Ruben, Coord. Chem. Rev. 2017, 346, 176.

[6] M. Cavallini, M. Melucci, ACS Appl. Mater. Interfaces 2015, 7, 16897.

[7] M. Sorai, S. Seki, J. Phys. Chem. Solids 1974, 35, 555.

[8] P. J. von Ranke, B. P. Alho, R. M. Ribas, E. P. Nobrega, A. Caldas, V. S. R. de Sousa, M. V. Colaço, L. F. Marques, D. L. Rocco, P. O. Ribeiro Phys. Rev. B 2018, 98, 224408.

[9] S. P. Vallone, A. N. Tantillo, A. M. dos Santos, J. J. Molaison, R Kulmaczewski, A. Chapoy, P. Ahmadi, M. A. Halcrow, K. G. Sandeman, Adv. Mater. 2019, 31, 1807334.

[10] I. Sarbu, C. Sebarchievici, Sustainability 2018, 10, 191.

[11] X. Perpiñà, M. Vellvehi, X. Jordà in Thermometry at the Nanoscale: Techniques and Selected Applications (Eds.: L. D. Carlos, F. Palacio), The Royal Society of Chemistry, 2016, pp. 383-436.

[12] Z. Ling, Z. Zhang, G. Shi, X. Fang, L. Wang, X. Gao, Y. Fang, T. Xu, S. Wang, X. Liu, Renew. Sust. Energ. Rev. 2014, 31, 427.

[13] C. Kinkelin, S. Lips, U. Soupremanien, V. Remondière, J. Dijon, H. Le Poche, E. Ollier, M. Zegaoui, N. Rolland, P.-A. Rolland, S. Lhostis, B. Descouts, Y. Kaplan, F. Lefèvre, Energy Convers. Manag. 2017, 142, 257.

[14] K. Pielichowska, K. Pielichowski, Prog. Mater. Sci. 2014, 65, 67.

[15] A. Sharma, V. V. Tyagi, C. R. Chen, D. Buddhi, Renew. Sust. Energ. Rev. 2009, 13, 318.

[16] A. Fallahi, G. Guldentops, M. Tao, S. Granados-Focil, S. Van Dessel, Appl. Therm. Eng. 2017, 127, 1427.

[17] O. Roubeau, M. Castro, R. Burriel, J. G. Haasnoot, J. Reedijk, J. Phys. Chem. B 2011, 115, 3003.

[18] S. Rat, K. Ridier, L. Vendier, G. Molnár, L. Salmon, A. Bousseksou, CrystEngComm 2017, 19, 3271.

[19] V. Shalabaeva, S. Rat, M. D. Manrique-Juarez, A.-C. Bas, L. Vendier, L. Salmon, G. Molnár, A. Bousseksou, J. Mater. Chem. C 2017, 5, 4419.

[20] K. Ridier, S. Rat, H. J. Shepherd, L. Salmon, W. Nicolazzi, G. Molnár, A Bousseksou, Phys. Rev. B 2017, 96, 134106.

[21] A.-C. Bas, X. Thompson, L. Salmon, C. Thibault, G. Molnár, O. Palamarciuc, L. Routaboul, A. Bousseksou, Magnetochemistry 2019, 5 28.

[22] W. P. Risk, C. T. Rettner, S. Raoux, Appl. Phys. Lett. 2009, 94, 101906

[23] C. M. Quintero, O. Kraieva, F. Carcenac, D. Lagrange, N. A. Yaremchuk, G. Molnár, C. Bergaud, Microelectron. J. 2015, 46, 1167.

[24] K. Ridier, A.-C. Bas, V. Shalabaeva, W. Nicolazzi, L. Salmon, G. Molnár A. Bousseksou, M. Lorenc, R. Bertoni, E. Collet, H. Cailleau, Adv. Mater. 2019, 31, 1901361.

[25] E. Ollier, U. Soupremanien, V. Remondière, J. Dijon, H. Le Poche, A. L. Seiler, F. Lefevre, S. Lips, C. Kinkelin, N. Rolland, P. A. Rolland, M. Zegaoui, S. Lhostis, P. Ancey, B. Descouts, Y. Kaplan, Microelectron. Eng. 2014, 127, 28.

[26] Y. Shi, C. Wang, Y. Yin, Y. Li, Y. Xing, J. Song, Adv. Funct. Mater. 2019 29, 1905470.

[27] E.-B. S. Mettawee, G. M. R. Assassa, Sol. Energy 2007, 81, 839.

[28] K. Lafdi, O. Mesalhy, S. Shaikh, J. Appl. Phys. 2007, 102, 083549. 


\section{Entry for the Table of Contents}

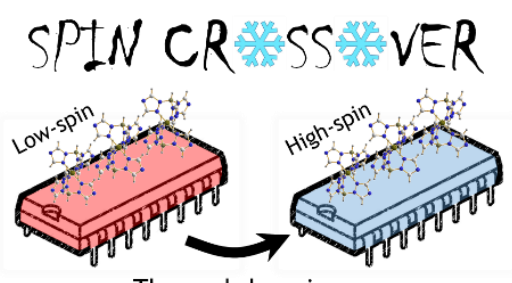

Thermal damping

Thermal management of metallic Joule-heated microwires was experimentally demonstrated using a molecular spin-crossover film as a thermal damper system. A transient attenuation of the wire temperature, up to $10 \%$, was evidenced due the excess heat capacity associated with the spin-state switching, providing a first milestone toward the assessment of the potential of spin-transition materials for thermal management applications. 